\title{
A Novel Image Retrieval Approach for Semantic Web
}

\author{
G. Nagarajan \\ Research Scholar Sathyabama University \\ Chennai,Tamilnadu, India
}

\author{
K.K Thyagharajan \\ Principal, RMK College of Engg \& Technology, \\ Chennai, Tamilnadu, India
}

\begin{abstract}
In this world of information technology revolution we human wants a way of automation in every field, but the intelligent technique behind the way how we visualize an image is not equivalent to how an computer system visualize .In web for a system the image is simply a resource with some specific hyper-link path. In this paper we propose an idea of giving semantic to a web image page so a system can understand the semantic behind the image which automatically increases the efficiency of image search. So we represent a way of converting an ordinary Syntactic page into a Semantic web page with corresponding Ontology which would pave the way of advancement in Semantic Web Learning technology.
\end{abstract}

\section{General Terms}

Image Retrieval and Semantic Web et. al.

\section{Keywords}

Web Crawler; NLP; SIFT; Ontology; Semantic Web

\section{INTRODUCTION}

The search for Knowledge is always indulged upon every human being. Even with the advancement of Artificial Intelligent still we may succeed in a way of building an rational agent but we can't build an agent which would think and act like human $[1,2,3]$. That's why most of the time Web image search engine provide you some of irrelevant image. The reason behind this slackness is the semantic gap of how an human eyes and brain visualize an image is not equivalent of how a machine visualize.

Though many researches have been proposed to fill those entire semantic gaps [4] the results are very much rationalized and inflexible. In this paper we propose a research of providing semantic to the web content and the web image so it can think and act like human. By providing semantic to the web page we are converting a ordinary syntactic page to an semantic web page which paves the way for Semantic Web Search Engine.

In this paper we are concentrating more on how to convert an ordinary page to a Semantic page. When comes to Semantic Web [3] the search engines are categories into Ontology Search Engine which search only for Ontology and Semantic Search Engine which uses the ontology for search. Due to the revolutionary idea behind the Semantic Web now a day's people start using these technologies in their corporate web pages for ease of usage. But still we have trillions of web page without semantic, so while if we design a Semantic Search Engine we can't avoid the content of those pages. So in this paper we are proposing an idea of converting the ordinary web pages to a Semantic Web page with respect to the image content, so that we can design an efficient Inter-operable Image Search Engine for Sematic Web which also takes account of the content of ordinary web pages. As shown in the Fig. 1 the proposal system consist of three modules. The Web intelligent module deals with the way of getting the intelligent information form the internet and it will the analyze and categorize the data. The Knowledge intelligent module deals with the way of getting the domain intelligent details from the human perceptive view. At last the Integration module integrate the intelligent details gather from the web and human which would give an effective semantic image retrieval system

This paper deals only with the web Intelligent module it is organized in such a way that in Section 2.1 explained how to gather the information from the web site using web crawler is been discussed in Section 2.2 the Web image analysis using SIFT is been discussed and in section 2.3 the Web page analysis using NLP is been discussed

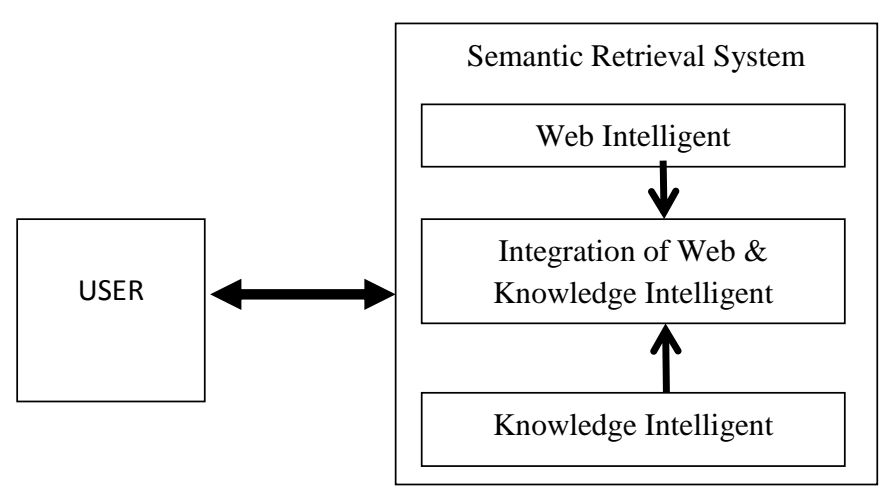

Figure1: Proposed Semantic Web intelligent Retrieval

\section{WEB INTELLIGENT}

Normally Intelligent is an abilities to learn from experience and to adapt new technique from challenging environment. Likewise Web Intelligent is also a way to deal with web content Fig. 2 shows it briefly.

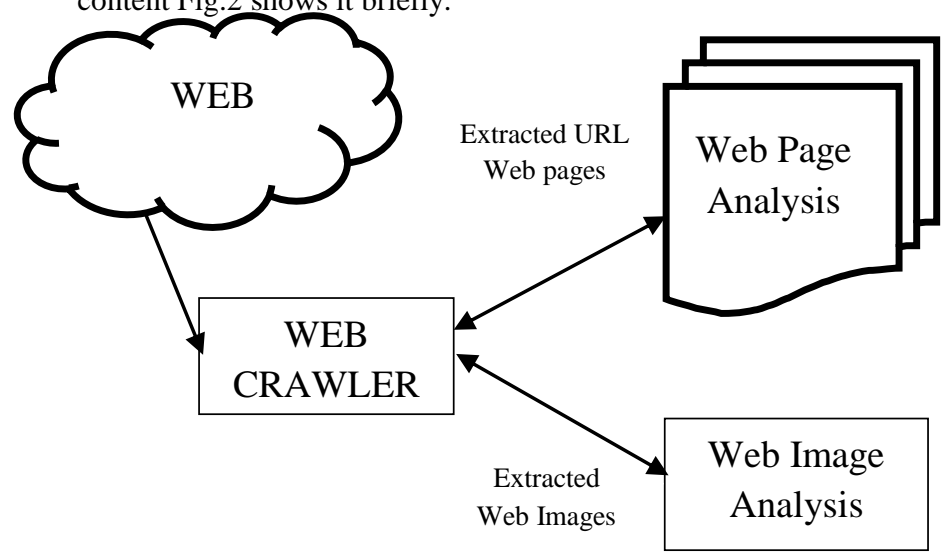

Figure 2: Web Intelligent 


\subsection{Web Crawler}

In this era of Information technology revolutionary World there are trillion of useful information in a Web but it was not in an organized way. What use if we propose an brilliant new idea through an journal paper which is not organized in an proper way. Such a way we are in need of complete organization in web pages so searching and knowledge acquisition would be efficient.

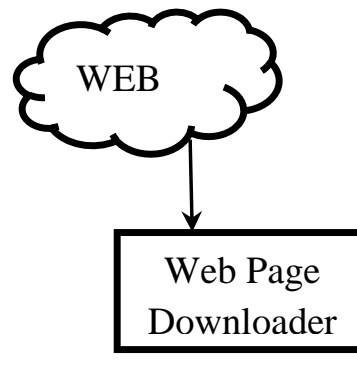

\subsection{Image Analysis}

In this image analysis stage we are going to analysis the extracted images from the Web Crawler to determine the knowledge behind each image that is identify the image automatically so as to match the concern domain .Fig 5 Shows briefly what are all the steps involved in this image analysis

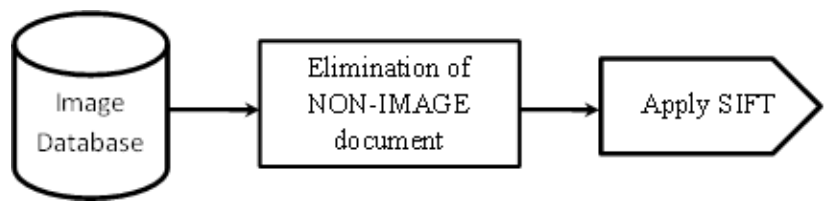

Figure 5: Proposed Image Analysis

Figure 3: Web Crawler design

So for Web based semantic search we should start from designing a Web Crawler. As shown in Fig 3 a Web Crawler is a simple browser program which would get the entire URL link from a web page. For all kind of Web Search Engine a Web Crawler plays an important role. It automatically gets and updates the list of URL which is usually called as Seeds [7] and store in an open source database.

As shown in the Fig 3 using a open source crawler program we can extract all kind of multimedia data. In this work we are going to extract only the page URL and the images on that particular webpage. Fig 4 shown the output for an given URL "www.Yahoo.com" the left-hand side shown the list of URL in that particular page and the right-hand side shows the collection of image extracted by an crawler for that particular web page. The way to analysis in order to acquisition knowledge form the web page is given below.

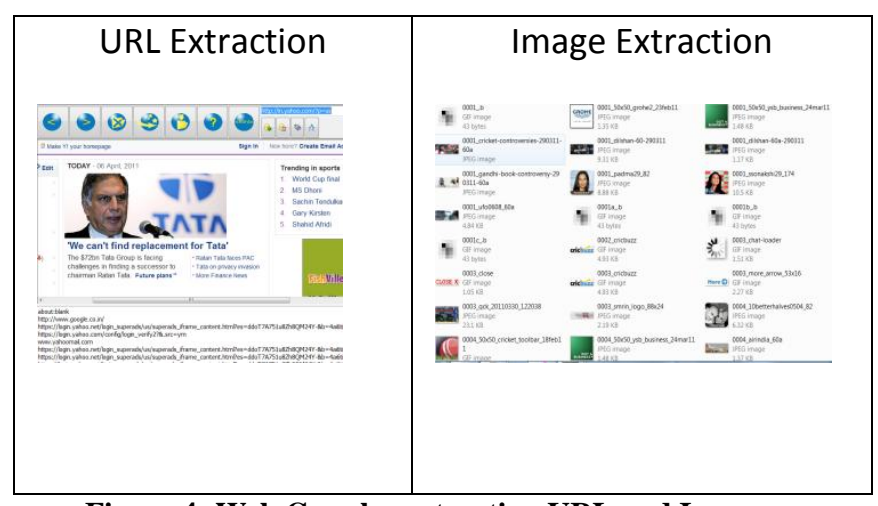

Figure 4: Web Crawler extracting URL and Image

\subsubsection{Determination of Image or Non ImageFile}

From Fig 4we can conclude that a web crawler simply get all the image files with the extension of .jpg, .gif, .bmp, .png and etc. So there are chances that an non image document would also be there in our image collection so first as shown in the Fig 5 we are going to eliminate those non images data by finding the Entropy of the images.

Entropy is used to measuring the uncertainty from a random variable. Here we can consider an image is random variable as they are random in nature because we cannot categorize an image.

$$
\text { Image Entropy }(I E)=-\sum\left[I * \log _{2}(I)\right]
$$

Where I, is the image histogram count. Usually we cannot find the Entropy value of color images. We have to convert it into a two dimension grey scale image and with that histogram count we can determine the entropy. Table 1 show the image Entropy for different image which is been collected by the Web crawler

From the table and with our experiment we conclude that an image with an entropy value greater than 7.2 is always a Non image as shown in the figure a "Yahoo" thumbnail image has the value of only 1.88 and so on.

IF IE $<=7.2$ Then $I=$ Image, Else $I=$ Non Image 


\subsubsection{Implementation of SIFT}

To search a data from a very large scale database is not a complicated task if the data is just a literal or numerical data. Since all those data has only one representation. If we have large collection of Image and to search an image from it by giving either image or text query as input is a very complicated job as an image can be represented in many different way with its different features. In this paper we have used SIFT [8][15] for image feature analysis as it is proved to provide better matching result [8] even there exist few many paper as an extension of SIFT such as PCA-SIFT[9] and ASIFT [9] still this is prove to be efficient to retrieve invariant and affine image when compare to the training image.

Table 1: Image Entropy

\begin{tabular}{|c|c|}
\hline Image & Entropy \\
\hline $\begin{array}{ll} & \text { 0003_close } \\
\text { CLOSE X } & \text { GIF image } \\
& 1.05 \mathrm{~KB}\end{array}$ & 4.95 \\
\hline $\begin{array}{l}\text { 0001_ssonakshi29_174 } \\
\text { JPEG image } \\
10.5 \mathrm{~KB}\end{array}$ & 7.423 \\
\hline $\begin{array}{l}\text { 0004_sachin0504_174 } \\
\text { JPEG image } \\
12.0 \mathrm{~KB}\end{array}$ & 7.44 \\
\hline $\begin{array}{r}\text { 0006_base } \\
\text { Yanoo: GIF image } \\
905 \text { bytes }\end{array}$ & 1.887 \\
\hline$\left(\begin{array}{l}0004 \text { _50r50_cricket_toolbar_18feb1 } \\
1 \\
\text { GIF image }\end{array}\right.$ & 5.4688 \\
\hline
\end{tabular}

Scale Invariant Feature Transform algorithm consist of four steps that is scale space extrema detection, key point localization, Orientation assignment and Key point description. Here we use SIFT to determine the key point and its corresponding orientation matrix so as to match with the trained image in Knowledge Intelligent phase.

\subsubsection{Scale Space Representation}

To determine the invariance feature at first the image is scaled using multi-scale signal representation concept. This is done by smoothing the image in different filter coefficient. The better filter suggest for smoothing is Gaussian filter as this is one of those filter which would not introduce any new spurious structure while filtering the image. A Gaussian function coefficient for an 2D data such as image is given by:

$$
G(x, y)=\frac{1}{2 \pi t} e^{-\left(\frac{(x-x 1)^{2}}{2 \sigma_{x}^{2}}-\frac{(y-y 1)^{2}}{2 \sigma_{y}^{2}}\right)}
$$

Now for an 2D image $f(x, y)$ its Gaussian scale space representation equation is given by

$$
L(x, y, t)=G(x, y, t) * f(x, y)
$$

Where the Gaussian filter value is convolute to the every single pixel value $f(x, y)$.
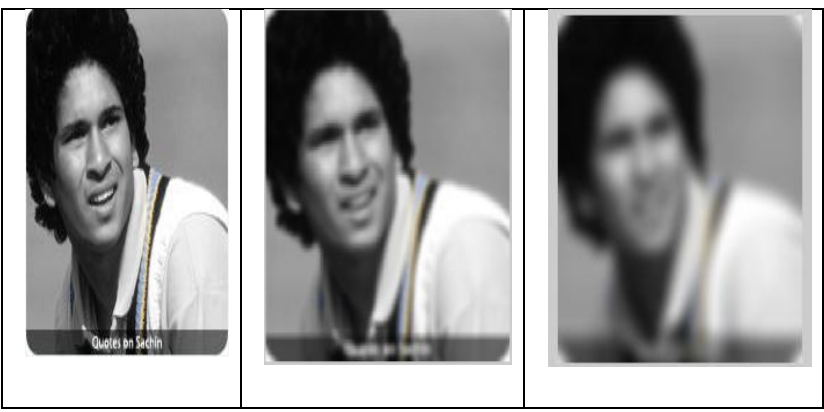

Figure 6: Image Smoothing by different Gaussian coefficient

To determine the key point in the image, an $\mathrm{n}$ number of Gaussian filtered image is taken the $\mathrm{n}$ should be an multiple of 5 as determined images are all grouped to 5 octave [8] normally an octave is said to be a interval between two different musical pitch which are half of its frequency, similarly here octave is an interval between two same images but with different filter coefficient

With this set of image the difference between the Gaussian filtered image is determine which is called as DoG Figure 7 shows how an two different Gaussian filtered image's DoG . The filter value of first image is [15x15] value of threshold of 15 and Second image is [15x15] value of threshold of .5. As shown the DoG image of all octaves is determined. From The Resultant number of image local extrema [10] is calculated as shown in Figure 8.

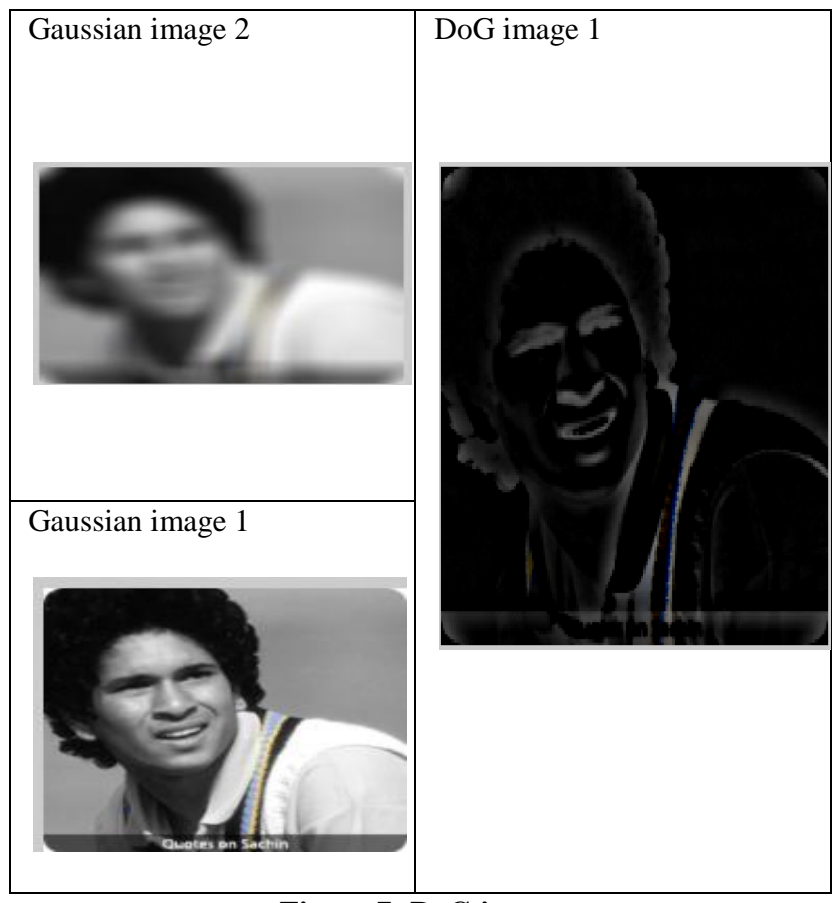

Figure 7: DoG image

An Local Extrema is used to find the maximum and minimum value of the given function within a given neighborhood and it is used to called as the maxima and minima, this kind of technique is basically used for optimization. 


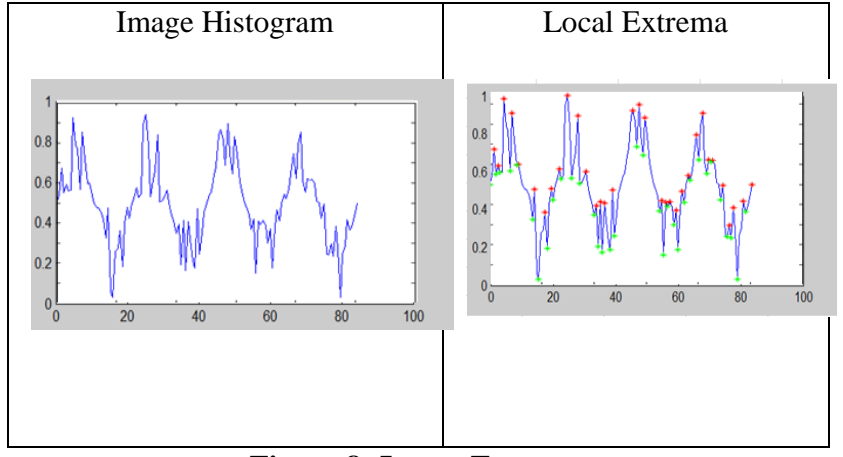

Figure 8: Image Extrema

\subsubsection{Key Point Detection}

Once we determine all the feature key point the number of key point would be numerous, if we process this much of key points for an single image the computation time would be more for comparing $\mathrm{N}$ images from different domain would be enormous so we can eliminate some of the key points which has low contract key point. This can be calculated by excluding the key point with local minima less than 0.03 or any other threshold can be also fixed. Also we can eliminate the key point near the edges this can be determine by determine the edges, in SIFT they have used Hessian Matrix for edge detection for eliminating the all those key point. In Figure 9 left hand side shows the key points without elimination and right hand side shows the key points those are eliminated using the above said strategy.

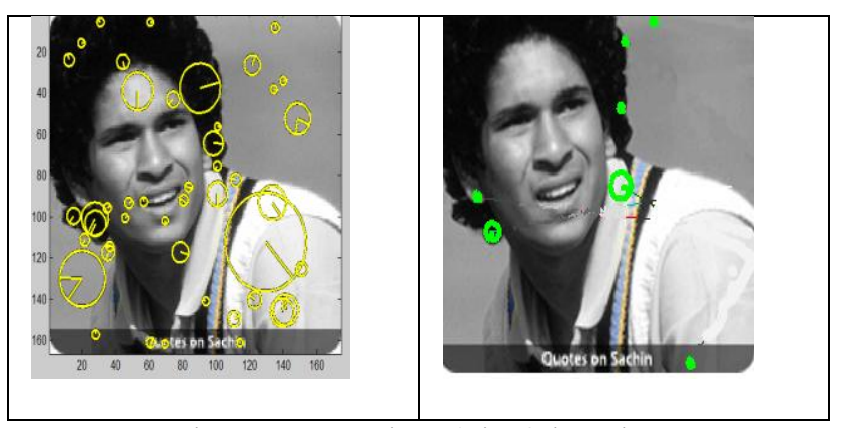

Figure 9: Key points \& its Orientation

\subsubsection{Orientation Detection}

From the collection of image as shown in Figure 3 we would determine the key feature points as shown in the figure 9. Also for all the key points generated the orientation of the key points are determined.

An image gradient is used to find the direction where an intensity of an image is transform from low intensity to high intensity. So with all these gradient detail an histogram is generated and those direction whose histogram value is maximum is taken as the concern key points orientation. This was also shown in Figure 9 with a line mark inside the circular key point.

\subsection{Web Page Analysis}

The main objective of this analysis it to find the domain of the web page (i.e whether the web page is belong to cricket or cartoons or any other genre of domain) once the domain of the web page is analyzed then we can index those web page and their corresponding image key points under one roof.
As shown in figure each and every web page is processed by the Natural Language Procedure. The reason [11] of using NLP is that as we use our own natural language for designing the web page we need a procedure which is mature enough to analysis human way of writing.

Here the web page content has to be converted to XML for this we use ESpotter [12,13,14] a NLP base tool which generates XML files for the inputted web document. This tool uses the Entity Name relationship concept for the XML creation. Such created XML has to be coverted to an UML diagram so that the relationship between each classes and sub classes can be determined and the repeated entity can be omitted. Such UML can be converted to RDF Schema, a semantic representation language .

\section{CONCLUSION}

If we have knowledge about what we are searching for, we can easy retrieve the desire information. The main drawback in information retrieval procedure in web technology is that the technology doesn't know the semantic and syntax of what the user searching for. This gives birth to the Semantic Web Technology. In this paper we have proposed an idea of retrieving the web images by providing semantic to it from the web content of the images. Here we designed a Semantic retrieving system using the web Intelligent. In our future work we will integrate the Web Intelligent with Knowledge Intelligent.

\section{REFERENCES}

[1] G.Nagarajan, Prof.K.K.Thyagharajan,"A Survey on the Ethical Implications of Semantic Web Technology": Journal of Advanced Reasearch in Computer Engineering,Vol.4No.1 jan-june 2010

[2] R.I.Minu,Prof.K.K.Thyagharajan,"Evolution of Semantic Web and Its Ontology": Second Conference on Digital Convergence 2009

[3] Yihun Alemu et al,"Image Retrieval in Multimedia Database:A Survey":Fifth Internation conference on intelligent information hiding and multimedia signal processing 2009

[4] Adolfazl Lakdashti,"Semantic- Based Image Retrieval: A Fuzzy Modeling Approach",IEEE conference 2008

[5] Yufei Li et al,"A Relation-Based Search Engine in Semantic Web": IEEE Transaction on Knowledge and Data Engineering, Vol 19,No.2,2007

[6] Gustavo Carneiro et al,"Supervised Learning of Semantic Classes for Image Annotion and Retrieval",IEEE Transcation on Pattern Analysis and Machine Intelligence,Vol:29,No:3,2007

[7] Hsien-Tsung Chang,"Web Image retrival systems with automatic web image annotaing techniques":WSEAS trancaction on Information science and application

[8] David G.Lowe,"Distinctive Image feature from scale Invariant Keypoints":International Journal of computer vision 2004

[9] Jean-Michel Morel and Guoshen Yu,"ASIFT:A New framework for fully affine invariant image comparison",SIAM J IMAGE ScIENCES Vol.2,No.2,2009. 
[10] Siddarth Jonathan JB et al ,"SQUINT SVM for identification of relevant sections in web page for web search": 2009 second international conference on intelligent computation technology and automation

[11] Diana Maynard et al, ,NLP techniques for term extration and ontology population"2008 conference on Ontology Learning and Population, Netherlands.

[12] Sabrina $T$ et al,"Extending ontology tree using NLP technique, In: Proceedings of National Conference on Research \& Development in Computer Science REDECS 2001.

[13] Juan D Velaquez and Jose I.Ferenandez," Towards the identification of important words from the web users point of view" International Workshop on Intelligent Web Based Tools (IWBT-07) in conjunction with 19th IEEE ICTAI-07,2007.

[14] Zhu,J.,Uren,V.\&Motta,E.,2005”,Espotter:Adaptive named entity recognition for web browsing". In Intelligent IT tools for knowledge Management System,KMTOOLS 2005.pp.518 - 529

[15] R.I.Minu and K.K.Thyagharajan "Automatic image classification using SVM Classifier"CiiT International Journal of Data Mining Knowledge Engineering,July issue, 2011

\section{AUTHORS PROFILE}

G. Nagarajan has received his Diploma in Electronic \& Communication Engineering from Directorate Of Technical Education 1997. He has received his BE degree in Electrical \& Electronic Engineering from Manonmaniam Sundaranar University 2000. He received his ME degree in Applied Electronic Engineering from Anna University 2005. He also received his ME degree in Computer Science Engineering from Sathyabama University 2007. He is at present a $\mathrm{PhD}$ Scholar in Computer Science Engineering from Sathyabama University. His research areas are web Image Mining, Artificial Intelligent, Ontology Learning, Machine Leaning, NLP and Semantic Web.

Dr. K.K. Thyagharajan has received his B.E., degree in Electrical and Electronics Engineering from PSG College of Technology (Madras University). He received his M.E., degree in Applied Electronics from Coimbatore Institute of Technology and Post Graduate Diploma in Computer Applications from Bharathiar University. He has received his Ph.D., (Multimedia Streaming) degree in Information and Communication Engineering from College of Engineering Guindy, Anna University. He has written 5 books in Computing. His book "Flash MX 2004" published by McGraw Hill (INDIA) has been recommended as text $/$ reference book by many universities. He has published more than 30 papers in National and International Journals and Conferences. He is a grant recipient of Tamil Nadu State Council for Science and Technology. His biography has been published in the 25th Anniversary Edition of Marquis Who's Who in the World Directory. He has been invited as chairperson and delivered special lectures in many National and International conferences and workshops. He is reviewer for many International Journals and Conferences. His current interests are Multimedia Networks, Mobile Computing, Web services, Data Mining, e-learning, Image Processing, Microprocessors and Microcontrollers. He has guided 10 M.E. projects and now 9 students are doing Ph.D. under him in the area of Multimedia, Image Processing and Data Mining. He is a life member of Computer Society of India and Chairman of the ISTE chapter of RMK College of Engineering and Technology. 